\title{
Diffusion-weighted Magnetic Resonance Imaging for Predicting Response to Chemoradiation Therapy for Head and Neck Squamous Cell Carcinoma: A Systematic Review
}

\author{
Sae Rom Chung, $M D^{1}$, Young Jun Choi, MD, PhD ${ }^{1}$, Chong Hyun Suh, MD ${ }^{1,2}$, Jeong Hyun Lee, MD, PhD ${ }^{1}$, \\ Jung Hwan Baek, MD, $\mathrm{PhD}^{1}$ \\ ${ }^{1}$ Department of Radiology and Research Institute of Radiology, University of Ulsan College of Medicine, Asan Medical Center, Seoul, Korea; \\ ${ }^{2}$ Department of Radiology, Namwon Medical Center, Namwon, Korea
}

Objective: To systematically review the evaluation of the diagnostic accuracy of pre-treatment apparent diffusion coefficient $(A D C)$ and change in $A D C$ during the intra- or post-treatment period, for the prediction of locoregional failure in patients with head and neck squamous cell carcinoma (HNSCC).

Materials and Methods: Ovid-MEDLINE and Embase databases were searched up to September 8, 2018, for studies on the use of diffusion-weighted magnetic resonance imaging for the prediction of locoregional treatment response in patients with HNSCC treated with chemoradiation or radiation therapy. Risk of bias was assessed by using the Quality Assessment Tool for Diagnostic Accuracy Studies-2.

Results: Twelve studies were included in the systematic review, and diagnostic accuracy assessment was performed using seven studies. High pre-treatment ADC showed inconsistent results with the tendency for locoregional failure, whereas all studies evaluating changes in $A D C$ showed consistent results of a lower rise in $A D C$ in patients with locoregional failure compared to those with locoregional control. The sensitivities and specificities of pre-treatment ADC and change in ADC for predicting locoregional failure were relatively high (range: $50-100 \%$ and $79-96 \%, 75-100 \%$ and $69-95 \%$, respectively). Metaanalytic pooling was not performed due to the apparent heterogeneity in these values.

Conclusion: High pre-treatment ADC and low rise in early intra-treatment or post-treatment ADC with chemoradiation, could be indicators of locoregional failure in patients with HNSCC. However, as the studies are few, heterogeneous, and at high risk for bias, the sensitivity and specificity of these parameters for predicting the treatment response are yet to be determined.

Keywords: Head and neck; Squamous cell carcinoma; Cancer; DWI; Diffusion-weighted imaging; Apparent diffusion coefficient; Prediction; Treatment response; Response assessment; Systematic review; Meta-analysis

\section{INTRODUCTION}

Patients with advanced-stage head and neck squamous

Received July 16, 2018; accepted after revision November 11, 2018.

Corresponding author: Young Jun Choi, MD, PhD, Department of Radiology and Research Institute of Radiology, University of Ulsan College of Medicine, Asan Medical Center, 88 Olympic-ro 43-gil, Songpa-gu, Seoul 05505, Korea.

- Tel: (822) 3010-1702 - Fax: (822) 476-0090

- E-mail: jehee23@gmail.com

This is an Open Access article distributed under the terms of the Creative Commons Attribution Non-Commercial License (https://creativecommons.org/licenses/by-nc/4.0) which permits unrestricted non-commercial use, distribution, and reproduction in any medium, provided the original work is properly cited. cell carcinoma (HNSCC) are mainly treated with the nonsurgical methods of concurrent chemoradiation therapy (CCRT) or radical radiotherapy to preserve organ function and maintain the quality of life $(1,2)$. Despite these rigorous treatment methods, treatment still fails at local or nodal sites in the head and neck in approximately $25-30 \%$ of patients (3-8). A reliable indicator for pre-treatment diagnosis of resistant HNSCC in patients could allow the CCRT regimes to be modified, or indicate the need for a switch to alternative strategies, improving their chances of success, and sparing the patients from ineffective treatment burdened by unnecessary toxicity (9). In addition, intratreatment scanning for the adaptation of radiotherapy fields to the changing size of the tumor is already under 
evaluation, providing an opportunity to monitor early treatment response and adjust CCRT regimes accordingly $(1,2,10,11)$. However, differentiation of residual cancer from post-treatment change using conventional magnetic resonance imaging (MRI), computed tomography (CT), 18-fluorodeoxyglucose positron emission tomography-CT $\left({ }^{18} \mathrm{~F}-\mathrm{FDG} \mathrm{PET} / \mathrm{CT}\right)$ in the early post-treatment period is a dilemma. The morphological criteria of CT and MRI with regard to volume regression (12-16), change in signal intensity $(17,18)$, and nodal density $(19,20)$ have shown heterogeneous results in the prediction of treatment response. The image results from ${ }^{18} \mathrm{~F}-\mathrm{FDG}$ PET/CT are also often suboptimal due to low spatial resolution and presence of treatment-induced inflammation during the first four months post CCRT that may be misleading (21). Endoscopy of primary sites could be hampered by radiation-induced mucositis. Biopsy of primary and nodal sites could be affected by sampling errors and may initiate superimposed infection, fail to heal, and cause worsening of complaints (22).

Diffusion-weighted imaging (DWI) is a functional MRI technique allowing the quantification of the diffusion of water molecules in a tumor by measuring the apparent diffusion coefficient (ADC). Recently, researchers have focused on DWI for predicting treatment response in patients with HNSCC, and it has been demonstrated that tumors with high $A D C$ values are less likely to respond to chemoradiation (23-32). This is probably because a high $A D C$ value may reflect the presence of micronecrosis, tumor hypoxia, high stromal content, and low cellularity (lower proliferation), which consequently increase the resistance to CCRT. In addition, the diagnostic accuracy of change in ADC values between the pre-treatment and early intra-treatment or post-treatment periods has been investigated for the prediction of treatment response, under the consideration that treatment with CCRT leads to cell death and reduction of restrictive barriers to diffusion, and therefore a consequent increase in the mean $\operatorname{ADC}$ value $(9,28,30,33$, 34). However, several conflicting results have been reported $(9,25,34-39)$, and previous studies have been limited by small numbers of patients and overlapping patient data $(22,29-32,34,40)$. To the best of our knowledge, no systematic review has assessed the role of DWI in predicting locoregional failure according to pre-treatment $A D C$ and change in $A D C$ during early intra-treatment or posttreatment in patients with HNSCC. Therefore, we performed this systematic review to evaluate the sensitivity and specificity of pre-treatment $A D C$ and change in $A D C$ during the early intra-treatment or post-treatment period, for the prediction of the locoregional response to definitive CCRT or radiation therapy in patients with HNSCC.

\section{MATERIALS AND METHODS}

\section{Literature Search Strategy}

A computerized search of the Ovid-MEDLINE and Embase databases was performed to identify relevant original articles on the use of DWI for the prediction of locoregional treatment response in patients with $\mathrm{HNSCC}$ treated with definitive CCRT or radiation therapy, up until September 8, 2018. The following search terms were used: [("head and neck") OR (oropharyngeal) OR (tongue) $O R$ (oral cavity) OR (oropharynx) OR (hypopharyngeal) OR (hypopharynx) OR (larynx) OR (laryngeal) OR (pharynx) OR (pharyngeal)] AND [(carcinoma) OR (carcinomas) OR (cancer) OR (cancers)] AND [(chemoradiation) OR (chemoradiotherapy) OR (radiotherapy) OR (radiation therapy)] AND [("diffusion weighted") OR ("diffusion-weighted") OR (dw-mri) OR (DWI) OR ("apparent diffusion coefficient") OR (ADC)]. Only studies published in English were included. The bibliographies of the selected articles were screened to identify other relevant articles.

\section{Inclusion Criteria}

Studies investigating the use of DWI for the prediction of locoregional treatment response in HNSCC were eligible for inclusion.

Studies or subsets of studies satisfying all of the following criteria were included:

1) Population: patients with histologically proven HNSCC who underwent definitive CCRT or radiation therapy.

2) Index test: imaging with MRI including DWI with provision of pre-treatment $A D C$ value or change in the pretreatment and early intra-treatment or post-treatment $A D C$ values.

3) Reference standard: the reference standards of the treatment outcome as determined by histologic confirmation or clinical/imaging follow-up, or a combination of these.

4) Outcomes: results of locoregional failure after definitive CCRT or radiation therapy, reported in sufficient detail.

5) Study design: all observational studies (retrospective or prospective). 


\section{Exclusion Criteria}

The exclusion criteria were as follows: 1) case reports, review articles, editorials, letters, comments, and conference proceedings; 2 ) studies with insufficient data on the locoregional failure and locoregional control; 3 ) studies that did not provide ADC values; 4) studies that monitored the intra-treatment response during CCRT; and 5) studies with overlapping patients and data. Two reviewers independently selected appropriate study reports using a standardized form.

\section{Data Extraction}

One reviewer extracted data from the studies with the second reviewer double-checking the accuracy of the extracted data and resolving any uncertainty through discussion. The following data were extracted from each of the selected studies onto standardized data forms:

1) Study characteristics: authors, year of publication, hospital or medical school, years of patient recruitment, sample size, and study design.

2) Demographic and clinical characteristics of patients: mean age, nodule size, and patient reference standards.

3) Imaging characteristics: timing of imaging, machine manufacturer and model, magnetic field strength, sequence, slice thickness, gap, and total acquisition time for DWI.

4) Interpretation: number of reviewers, experience, presence of consensus data.

5) True positives and negatives and false positives and negatives for the prediction of treatment response according to ADC value. In cases of incomplete $2 \times 2$ tables, the corresponding author was contacted, and data required to generate $2 \times 2$ tables was requested.

\section{Quality Assessment}

The methodological quality of the included studies was assessed independently by two reviewers using tailored questionnaires devised according to the Quality Assessment of Diagnostic Accuracy Studies-2 (QUADAS-2) criteria (41). Disagreements were very minor and were resolved by consensus.

\section{Data Analysis}

For the diagnostic accuracy assessment, $2 \times 2$ data were summarized in forest plots of sensitivity and specificity for each study. Pooling was not performed because of the relatively small number of studies, relatively high risk of bias, and inherent heterogeneity based on varying study designs among the included studies. The presence of a threshold effect was visually assessed using coupled forest plots of sensitivity and specificity. The Spearman correlation coefficient between the sensitivity and false-positive rate was obtained; a value $>0.6$ was deemed a considerable threshold effect (42).

\section{RESULTS}

\section{Literature Search}

The study selection process is illustrated in Figure 1. Twelve studies were included in the systematic review and seven of these presented data that could be extracted to a $2 \times 2$ table format to calculate sensitivity and specificity. The seven studies included four investigating the diagnostic accuracy of pre-treatment $\operatorname{ADC}(25,26,28,30)$ for predicting the locoregional treatment response, and four investigating the diagnostic accuracy of change in $\operatorname{ADC}(9,25,33,34)$.

\section{Characteristics of the Included Studies}

The characteristics of the 12 included studies are listed in Table 1 . The 12 original articles included ten prospective studies $(9,24,25,30,33-38)$, one retrospective study (26), and one study with an unclear design (28). The target lesions of the studies were primary tumors $(n=3)(26$, $30,34)$, lymph nodes $(n=4)(24,25,28,37)$, or both $(n=5)(9,33,35,36,38)$. Eleven studies investigated the value of pre-treatment $\operatorname{ADC}(9,24-26,28,30,34-38)$ for predicting locoregional treatment response, and ten studies investigated the value of change in $\operatorname{ADC}(9,25$, $28,30,33-38)$. All studies had a clear description of the reference standard for determining the treatment outcome, and detailed descriptions of the proportion of patients with locoregional failures and locoregional control. Among the total population of 418 patients, locoregional failure occurred in 136 patients (32.5\%) and locoregional control was achieved in 282 patients (67.5\%) (analysis per-patient).

All studies included pre-treatment MRI including DWI, and ten studies performed MRI at early intra-treatment or posttreatment periods, to evaluate the change in ADC value (9, $25,28,30,33-38)$. The detailed technical characteristics of the MRI acquisitions are listed in Table 2. All of the DWI images were acquired in the axial plane using spin-echo echo-planar imaging sequences. The number of b-values used for the DWI ranged from one to ten, with majority of the studies using b-values between one and six $(9,24-26$, $28,30,33-38$ ). The regions of interest (ROIs) were outlined 


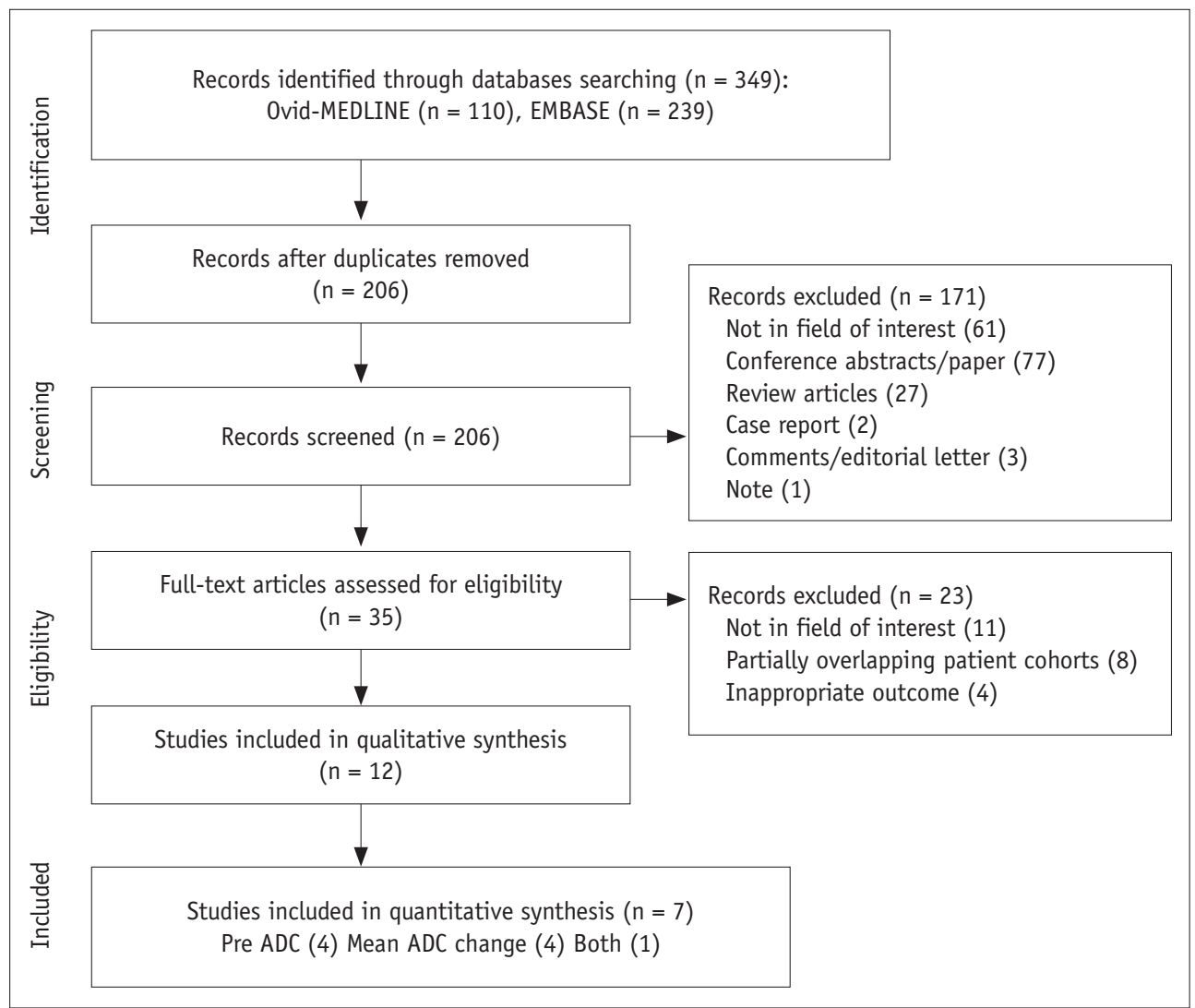

Fig. 1. Flow diagram of study selection process. $A D C=$ apparent diffusion coefficient

by experienced radiologists or oncologists, and were defined on either a single slice of the target lesion $(24,26,30,35$ $37)$, or on every section of all targeted lesions $(9,25,28$, $34,38,43)$.

\section{Quality Assessment}

Overall, the quality of the studies was considered moderate, with 9 of the 12 studies satisfying at least 5 of the 7 QUADAS-2 domains (Fig. 2). Notable areas of quality concerns included no mention of blinding to the clinical outcomes of patients during ROI placement (24-26, 28, $34,35,37,38)$. Regarding the patient selection domain, one study was considered to have a high risk of bias due to a non-consecutive case/control study design (26). In addition, one study was considered to have an unclear risk of bias as it did not explicitly mention whether patient enrollment was prospective or not (28). Only one study had a concern for applicability, which was because majority of the included patients were tested positive for human papillomavirus (HPV) (37). With regard to the reference standard and flow and timing domain, all studies were considered to have a low risk of bias.
Pre-treatment ADC Value for Predicting Locoregional Treatment Response of HNSCC

Eleven studies investigated the value of pre-treatment ADC for predicting locoregional treatment response in patients with $\operatorname{HNSCC}(9,24-26,28,30,34-38)$. Of these 11 studies, 5 found that pre-treatment ADC values were significantly associated with locoregional treatment response (24-26, 28,30 ); in all 5 of these studies, pre-treatment $A D C$ values were significantly higher in patients with locoregional failure than in those with locoregional control. A cut-off $A D C$ value ranging from 0.86 to 1.2 was mentioned in 4 studies $(25,26,28,30)$. In the other 6 studies that did not show a significant difference in ADC between the 2 groups, 2 studies showed lower values of pre-treatment ADC in locoregional control $(9,34)$, whereas in 3 studies, the pre-treatment $A D C$ value was higher in the locoregional control (36-38). In 1 study, locoregional control showed higher $A D C$ values than locoregional failure in primary tumors, but showed lower ADC values in lymph nodes (35). The diagnostic accuracy of pre-treatment ADC values for predicting locoregional failure was assessed in 4 studies $(25,26,28,30)$. Figure 3 is a forest plot of sensitivity and 


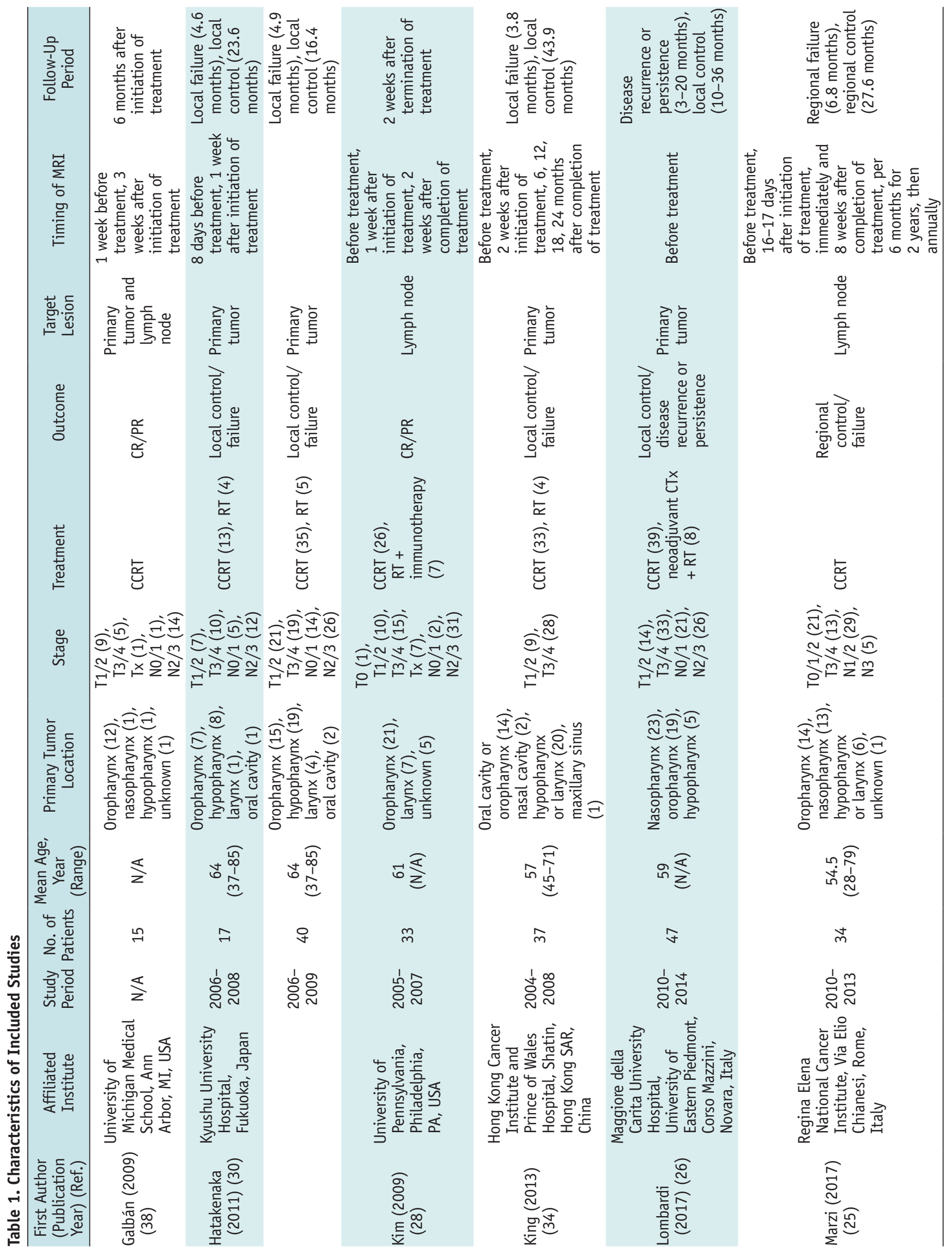




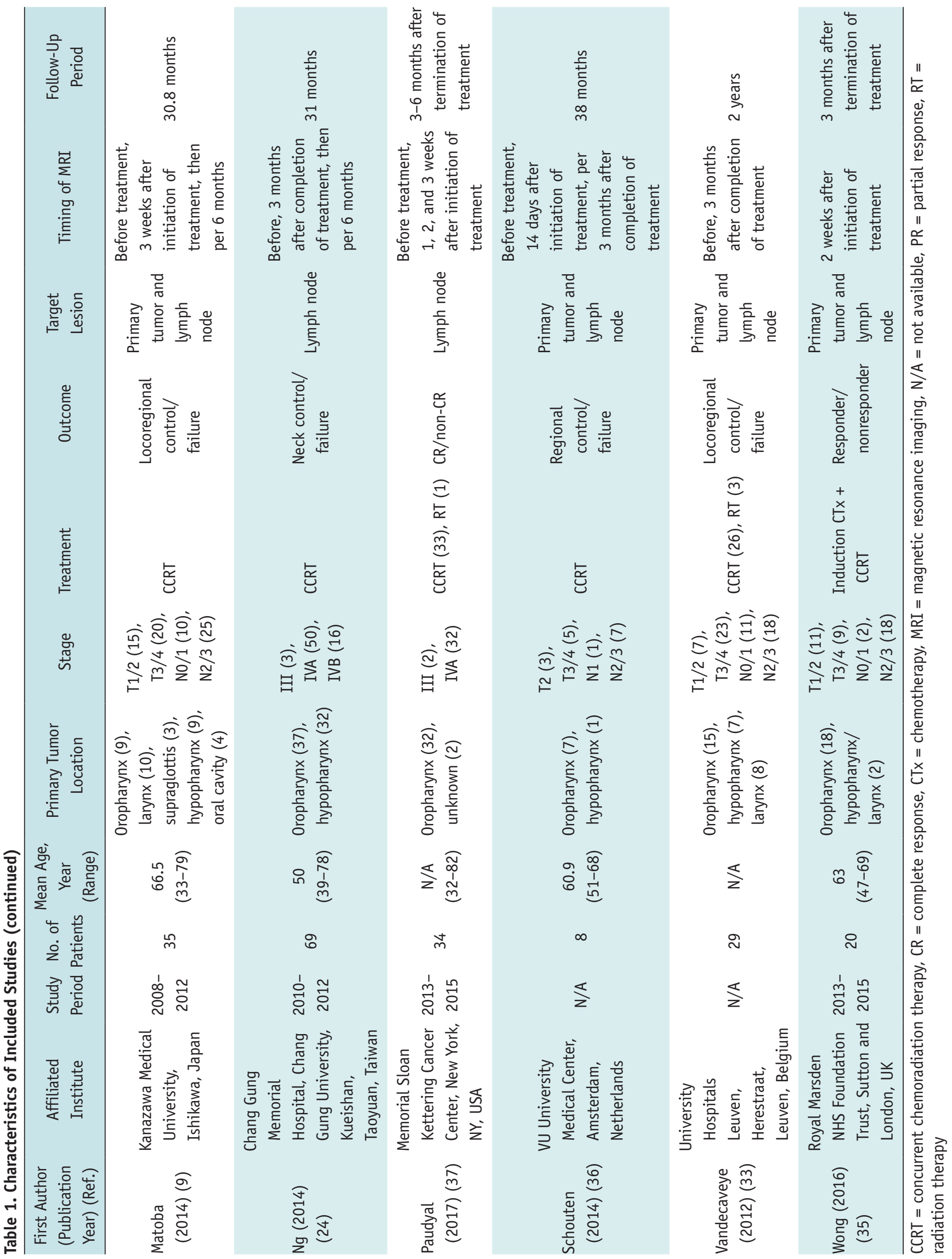




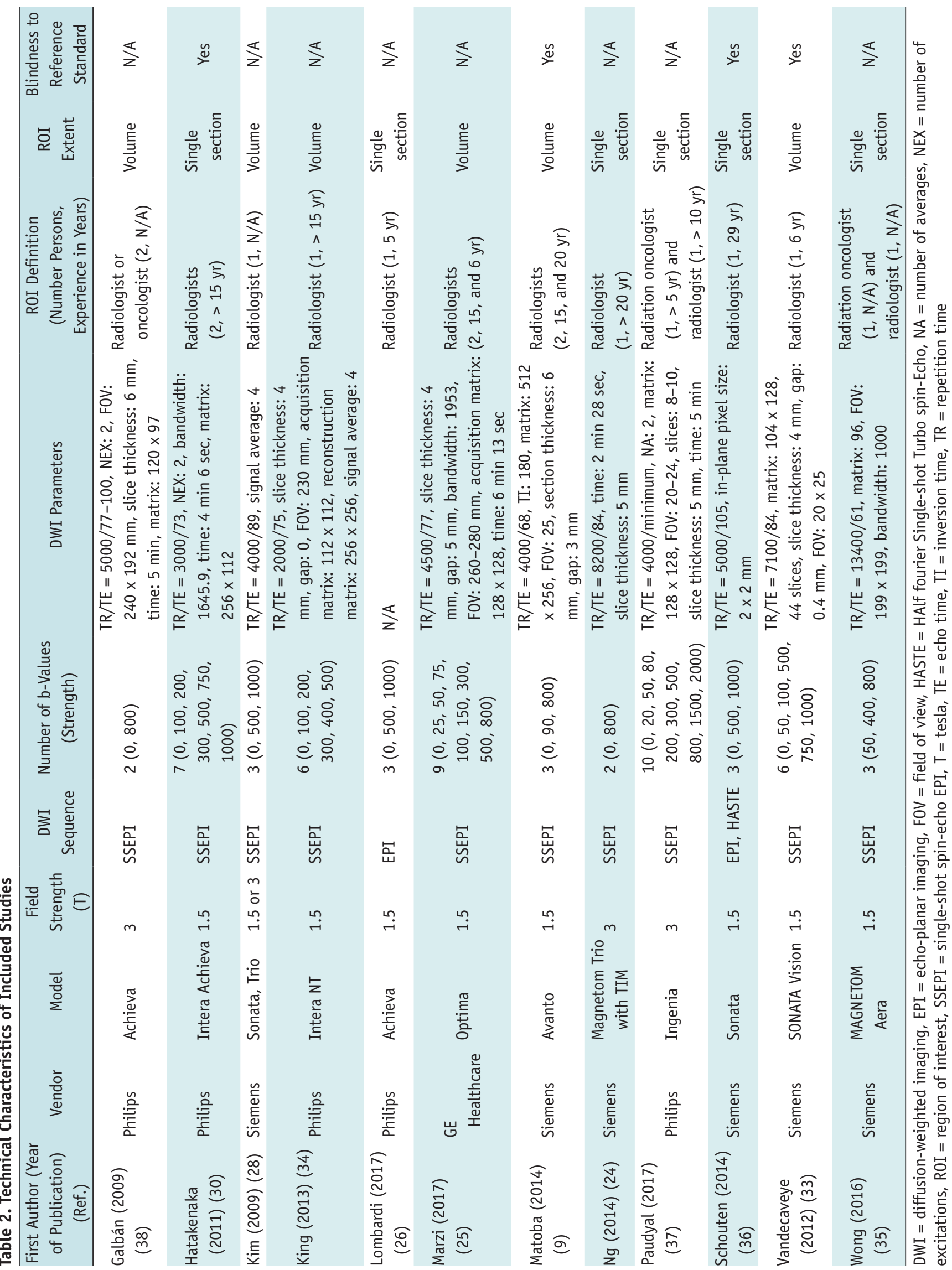




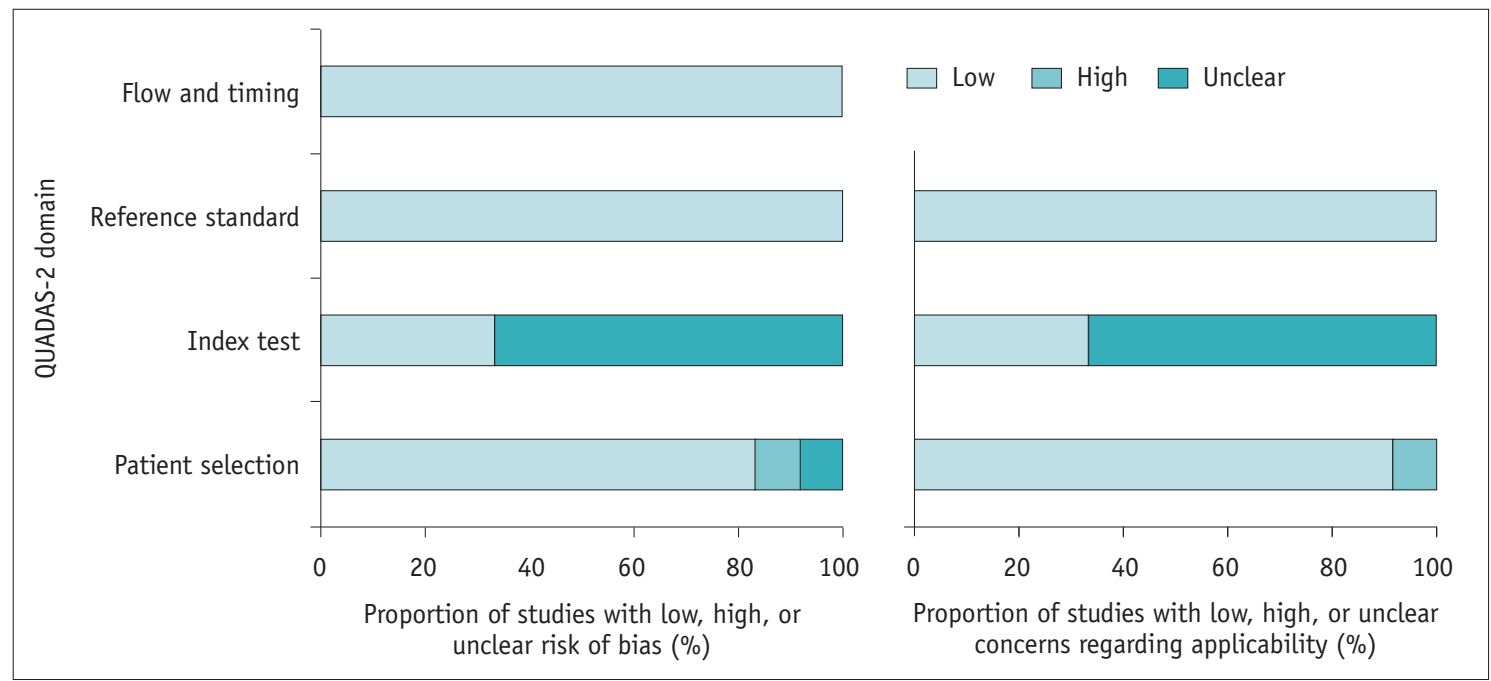

Fig. 2. QUADAS-2 criteria for included studies. QUADAS-2 = Quality Assessment of Diagnostic Accuracy Studies-2

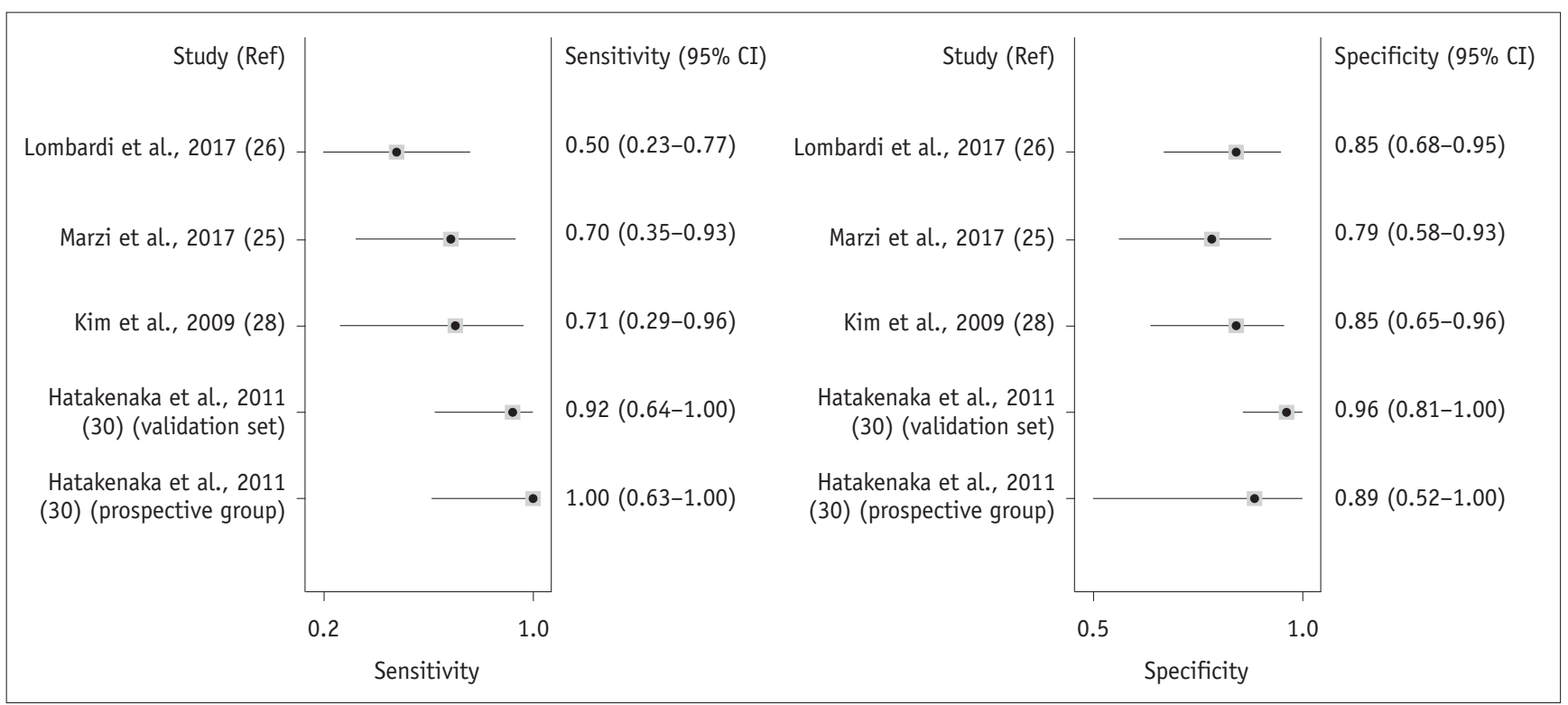

Fig. 3. Forest plots of sensitivity and specificity of pre-treatment ADC for prediction of locoregional recurrence. Horizontal lines indicate $95 \%$ CIs of individual studies. CI = confidence interval, Ref $=$ reference

specificity for the 4 included studies. The coupled forest plots of the sensitivity and specificity for the pre-treatment ADC did not reveal any apparent threshold effect and the Spearman correlation coefficient between sensitivity and false-positive rate was -0.519 ( $95 \%$ confidence interval [CI], -0.961-0.670). Meta-analytic pooling of the sensitivity and specificity values was not performed due to the apparent heterogeneity in these values that were unexplainable with threshold effect, and infeasibility of robust analysis of the causes of heterogeneity. The total population comprised of 171 patients, with 52 patients $(30.4 \%)$ having locoregional failure. The sensitivities and specificities of the 4 individual studies ranged from $50 \%$ to $100 \%$ and from $79 \%$ to $96 \%$, respectively (Table 3 ).

\section{Change in ADC Value for Predicting Locoregional Failure} of HNSCC

Ten studies investigated the value of change in $A D C$ for predicting locoregional treatment response in $\operatorname{HNSCC}(9$, $25,28,30,33-38)$. In all 10 studies, the change in ADC was larger in the patients with locoregional control than in those with locoregional failure, and 5 studies showed a statistically significant difference $(9,28,30,33,34)$. The cut-off value for change in $A D C$ was mentioned in 4 
Table 3. Summary of Diagnostic Accuracy of Pre-Treatment ADC Value in Predicting Locoregional Failure in HNSCC

\begin{tabular}{lrrrrrrc}
\hline \multicolumn{1}{c}{ Study } & Total & TP & FP & FN & TN & Sensitivity & Specificity \\
\hline Hatakenaka et al., 2011 (30) (prospective group) & 17 & 8 & 1 & 0 & 8 & 1.00 & 0.89 \\
Hatakenaka et al., 2011 (30) (validation set) & 40 & 12 & 1 & 1 & 26 & 0.92 & 0.96 \\
Kim et al., 2009 (28) & 33 & 5 & 4 & 2 & 22 & 0.71 & 0.85 \\
Marzi et al., 2017 (25) & 34 & 7 & 5 & 3 & 19 & 0.70 & 0.79 \\
Lombardi et al., 2017 (26) & 47 & 7 & 5 & 7 & 28 & 0.50 & 0.85 \\
\hline
\end{tabular}

$A D C=$ apparent diffusion coefficient, $\mathrm{FN}=$ false negative, $\mathrm{FP}=$ false positive, $\mathrm{HNSCC}=$ head and neck squamous cell carcinoma, $\mathrm{TN}=$ true negative, TP = true positive

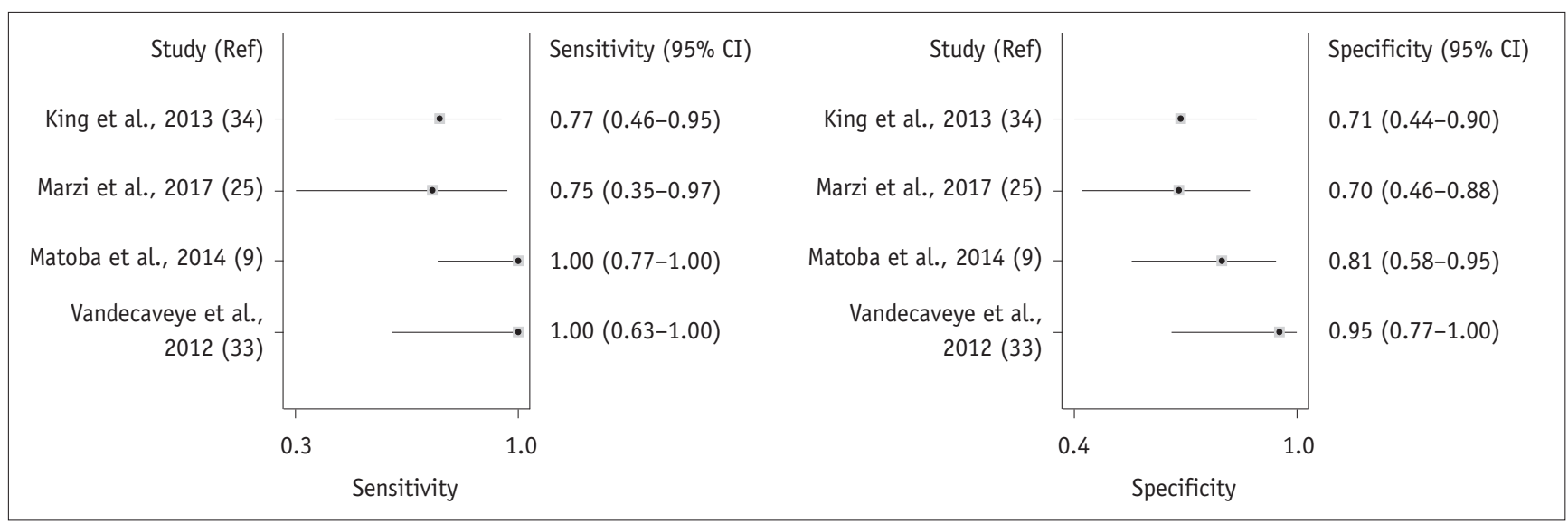

Fig. 4. Forest plots of sensitivity and specificity of change in ADC for prediction of locoregional recurrence. Horizontal lines indicate $95 \%$ CIs of individual studies.

Table 4. Summary of Diagnostic Accuracy of Change in ADC Value in Predicting Locoregional Failure in HNSCC

\begin{tabular}{lcrrrrrr}
\hline \multicolumn{1}{c}{ Study } & Total & TP & FP & FN & TN & Sensitivity & Specificity \\
\hline Vandecaveye et al., 2012 (33) & 30 & 8 & 1 & 0 & 21 & 1.00 & 0.95 \\
Matoba et al., 2014 (9) & 35 & 14 & 4 & 0 & 17 & 1.00 & 0.81 \\
Marzi et al., 2017 (25) & 28 & 6 & 6 & 2 & 14 & 0.75 & 0.70 \\
King et al., 2013 (34) & 30 & 10 & 5 & 3 & 12 & 0.77 & 0.71 \\
\hline
\end{tabular}

studies, and ranged from $15.5 \%$ to $25 \%(9,25,33,34)$. The time over which the change in $A D C$ was measured ranged from one to three weeks from the start of CCRT, except for one study where the ADC was measured three weeks after completion of CCRT (33). The diagnostic accuracy of the change in $A D C$ for predicting locoregional failure was assessed using 4 studies $(9,25,33,34)$. The coupled forest plots of sensitivity and specificity for the change in ADC did not reveal any apparent threshold effect and the Spearman correlation coefficient between sensitivity and false-positive rate was -0.829 (95\% CI, -0.996-0.650). (Fig. 4). Metaanalytic pooling of the sensitivity and specificity values was not performed for the same reasons as those mentioned above. The total population comprised of 135 patients, with locoregional failure in $43(31.9 \%)$. The sensitivities and specificities of the 4 individual studies ranged from $75 \%$ to $100 \%$ and from $69 \%$ to $95 \%$, respectively (Table 4 ).

\section{DISCUSSION}

In the current systematic review, we demonstrated that high pre-treatment $A D C$ and a low rise in $A D C$ during the early intra-treatment or post-treatment periods of CCRT were indicators of locoregional failure in patients with HNSCC. Considering the consistency in the results of change in $A D C$ obtained, we propose that it could be a promising approach to predict treatment response after CCRT.

In clinical practice, an accurate prediction of disease progression after treatment could be extremely useful for selecting the appropriate adjuvant treatment and improving the patient's prognosis (9). Cases of HNSCCs with high stromal content, low cellularity, and micronecrosis are associated with resistance to treatment and poor outcome (44). These tumor characteristics decrease diffusion of water molecules (45-47), and therefore it is 
hypothesized that high $A D C$ is a predictor of poor outcome. However, the results from using pre-treatment ADC for the prediction of locoregional failure are inconsistent. The treatment response may be attributed to differences in tumor aggressiveness, HPV status, treatment protocol, or the intensity of treatment, and hence, the use of only a single $A D C$ measurement at pre-treatment appears to be inadequate for the prediction of treatment response $(9,35)$.

As response-adapted therapy becomes more widespread in cancer management, there will be greater interest in performing intra-treatment scanning (44). Increase in ADC during treatment has been correlated with the histological presence of necrosis, apoptosis, and inflammation (33, 48), and is thought to be a useful predictor of treatment response. All of the 10 studies that investigated the role of change in $A D C$ at early intra-treatment or post-treatment periods found consistent results $(9,25,28,30,33-38)$. A lower rise in the mean $A D C$ was found at one to three weeks after the start of treatment in patients with locoregional failure, compared to that in patients with locoregional control. This approach may be more appropriate because the change in $A D C$ is more objective and reproducible across centers than absolute $A D C$ values (i.e., pre-treatment $A D C$ ) (44). In many malignant tumors, it is well known that successful treatment is correlated with an increase in $A D C$ values (49-53). Additionally, the ability of DWI to predict treatment outcome at one to three weeks after the start of treatment seems to be in agreement with the expected optimal timing of adjuvant neck dissection after CCRT or radiotherapy (54-56).

Heterogeneity was not quantified since it is an expected flaw in systematic reviews of diagnostic test accuracy. Instead, the possible sources for heterogeneity were explored. First, among the 12 studies, 3 acquired data from the primary tumors $(26,30,34), 4$ from lymph nodes $(24,25,28,37)$, and 5 from both sites $(9,33,35,36$, 38). The study by Wong et al. (35) found that mean pretreatment $A D C$ values were higher in locoregional control than in locoregional failure in primary tumors, but found opposite results in lymph nodes. It may be related to the fact that $A D C$ values acquired from the primary sites may be more influenced by physiologic motion and susceptibility artifacts than those acquired from cervical lymph nodes (9). Second, the included studies used different numbers and distributions of b-values, with majority of the studies using one to six b-values $(9,24,26,28,30,33-36,38)$, although 2 studies used nine or ten b-values $(25,37)$.
Recent studies show that mean ADCs obtained from high b-value ranges of $300-1000 \mathrm{~s} / \mathrm{mm}^{2}$ are more appropriate for predicting treatment response than mean $A D C s$ obtained from low b-value ranges of $0-300 \mathrm{~s} / \mathrm{mm}^{2}(27,30,44,57)$. Finally, the HNSCC showed heterogeneous histopathology with areas of micronecrosis, even though it was not readily distinguishable on imaging. Therefore, the use of the mean $A D C$ of the whole tumor is considered more accurate than the measurement from a single section ROI (9).

The prediction of tumor response with $A D C$ offers several advantages over the use of other imaging modalities like ${ }^{18} \mathrm{~F}$-FDG PET/CT, contrast-enhanced MRI, magnetic resonance spectroscopy, and dynamic contrast-enhanced MRI. These include: absence of the need for injection of an isotope or contrast agent, short acquisition time, and simple estimation. Despite these advantages of $A D C$, the clinical use of $A D C$ for the prediction of tumor response in HNSCC presents challenges due to susceptibility and motion artifacts. Furthermore, there is no clear threshold for the differentiation between locoregional failure and locoregional control. Finally, different MRI systems and different $b$-values have been used in previous studies. This means that the use of ADC cannot be extrapolated across hospital sites. Thus, further clinical studies to standardize and validate $A D C$ measurements are necessary.

This study was limited by the relatively few included studies and its potential heterogeneity. This precluded our ability to perform meta-analysis, analyze subgroups, and identify potentially important covariates. When sufficient papers have been published in the future, a meta- analysis considering the factors that may cause heterogeneity may be performed.

In conclusion, high pre-treatment $A D C$ and a low rise in $A D C$ during the early intra-treatment or post-treatment periods of CCRT could be indicators of locoregional failure in patients with HNSCC. Considering the consistency of the results obtained with change in $A D C$, we propose that it could be used to identify patients who require more aggressive investigations to identify any residual cancer. However, as the studies are few, heterogeneous, and at high risk for bias, the sensitivity and specificity of these parameters for predicting treatment response are yet to be determined. Continued research on standardization and validation of $A D C$ measurement, and determination of the optimal threshold for percentage change, are required for clinical use. 


\section{Conflicts of Interest}

The authors have no potential conflicts of interest to disclose.

\section{ORCID iDs}

Young Jun Choi

https://orcid.org/0000-0001-7098-5042

Sae Rom Chung

https://orcid.org/0000-0003-4219-7166

\section{REFERENCES}

1. Bonner JA, Harari PM, Giralt J, Azarnia N, Shin DM, Cohen RB, et al. Radiotherapy plus cetuximab for squamous-cell carcinoma of the head and neck. N Engl J Med 2006;354:567-578

2. Argiris A, Karamouzis MV, Raben D, Ferris RL. Head and neck cancer. Lancet 2008;371:1695-1709

3. Chiesa F, Mauri S, Tradati N, Calabrese L, Giugliano G, Ansarin $M$, et al. Surfing prognostic factors in head and neck cancer at the millennium. Oral Oncol 1999;35:590-596

4. Ang KK, Trotti A, Brown BW, Garden AS, Foote RL, Morrison WH, et al. Randomized trial addressing risk features and time factors of surgery plus radiotherapy in advanced head-andneck cancer. Int J Radiat Oncol Biol Phys 2001;51:571-578

5. Agra IM, Carvalho AL, Ulbrich FS, de Campos OD, Martins EP, Magrin J, et al. Prognostic factors in salvage surgery for recurrent oral and oropharyngeal cancer. Head Neck 2006;28:107-113

6. Carvalho AL, Magrin J, Kowalski LP. Sites of recurrence in oral and oropharyngeal cancers according to the treatment approach. Oral Dis 2003;9:112-118

7. Goodwin WJ Jr. Salvage surgery for patients with recurrent squamous cell carcinoma of the upper aerodigestive tract: when do the ends justify the means? Laryngoscope 2000;110:1-18

8. Kowalski LP, Bagietto R, Lara JR, Santos RL, Silva JF Jr, Magrin $\mathrm{J}$. Prognostic significance of the distribution of neck node metastasis from oral carcinoma. Head Neck 2000;22:207-214

9. Matoba M, Tuji H, Shimode Y, Toyoda I, Kuginuki Y, Miwa K, et al. Fractional change in apparent diffusion coefficient as an imaging biomarker for predicting treatment response in head and neck cancer treated with chemoradiotherapy. AJNR Am J Neuroradiol 2014;35:379-385

10. Newman JP, Terris DJ, Pinto HA, Fee WE Jr, Goode RL, Goffinet DR. Surgical morbidity of neck dissection after chemoradiotherapy in advanced head and neck cancer. Ann Otol Rhinol Laryngol 1997;106:117-122

11. Lavertu P, Bonafede JP, Adelstein DJ, Saxton JP, Strome M, Wanamaker JR, et al. Comparison of surgical complications after organ-preservation therapy in patients with stage III or IV squamous cell head and neck cancer. Arch Otolaryngol Head Neck Surg 1998;124:401-406
12. Davuluri R, Krase JM, Cui H, Goyal UD, Cheung MK, Hsu CC, et al. Image guided volumetric response during chemoradiotherapy for head and neck squamous cell carcinoma as a predictor of disease outcomes. Am J Otolaryngol 2016;37:304-310

13. Tang C, Fuller CD, Garden AS, Awan MJ, Colen RR, Morrison WH, et al. Characteristics and kinetics of cervical lymph node regression after radiation therapy for human papillomavirusassociated oropharyngeal carcinoma: quantitative image analysis of post-radiotherapy response. Oral Oncol 2015;51:195-201

14. Belli ML, Fiorino C, Zerbetto F, Raso R, Broggi S, Chiara A, et al. Early volume variation of positive lymph nodes assessed by in-room mega voltage CT images predicts risk of loco-regional relapses in head and neck cancer patients treated with intensity-modulated radiotherapy. Acta Oncol 2015;54:14901495

15. Ojiri $\mathrm{H}$, Mendenhall WM, Mancuso AA. CT findings at the primary site of oropharyngeal squamous cell carcinoma within 6-8 weeks after definitive radiotherapy as predictors of primary site control. Int J Radiat Oncol Biol Phys 2002;52:748-754

16. Hermans R, Pameijer FA, Mancuso AA, Parsons JT, Mendenhall WM. Laryngeal or hypopharyngeal squamous cell carcinoma: can follow-up CT after definitive radiation therapy be used to detect local failure earlier than clinical examination alone? Radiology 2000;214:683-687

17. King AD, Keung CK, Yu KH, Mo FK, Bhatia KS, Yeung DK, et al. T2-weighted MR imaging early after chemoradiotherapy to evaluate treatment response in head and neck squamous cell carcinoma. AJNR Am J Neuroradiol 2013;34:1237-1241

18. Lell M, Baum U, Greess H, Nomayr A, Nkenke E, Koester M, et al. Head and neck tumors: imaging recurrent tumor and post-therapeutic changes with CT and MRI. Eur J Radiol 2000;33:239-247

19. Sanguineti G, Ricchetti F, Wu B, Agrawal N, Gourin C, Agbahiwe $\mathrm{H}$, et al. Volumetric change of human papillomavirus-related neck lymph nodes before, during, and shortly after intensitymodulated radiation therapy. Head Neck 2012;34:1640-1647

20. Munck JN, Cvitkovic E, Piekarski JD, Benhamou E, Recondo G, Bachouchi M, et al. Computed tomographic density of metastatic lymph nodes as a treatment-related prognostic factor in advanced head and neck cancer. J Natl Cancer Inst 1991;83:569-575

21. McCollum AD, Burrell SC, Haddad RI, Norris CM, Tishler $R B$, Case MA, et al. Positron emission tomography with $18 \mathrm{~F}$-fluorodeoxyglucose to predict pathologic response after induction chemotherapy and definitive chemoradiotherapy in head and neck cancer. Head Neck 2004;26:890-896

22. King AD, Mo FK, Yu KH, Yeung DK, Zhou H, Bhatia KS, et al. Squamous cell carcinoma of the head and neck: diffusionweighted MR imaging for prediction and monitoring of treatment response. Eur Radiol 2010;20:2213-2220

23. Srinivasan A, Chenevert TL, Dwamena BA, Eisbruch A, Watcharotone K, Myles JD, et al. Utility of pretreatment mean apparent diffusion coefficient and apparent diffusion 
coefficient histograms in prediction of outcome to chemoradiation in head and neck squamous cell carcinoma. J Comput Assist Tomogr 2012;36:131-137

24. Ng SH, Lin CY, Chan SC, Lin YC, Yen TC, Liao CT, et al. Clinical utility of multimodality imaging with dynamic contrastenhanced MRI, diffusion-weighted MRI, and 18F-FDG PET/ CT for the prediction of neck control in oropharyngeal or hypopharyngeal squamous cell carcinoma treated with chemoradiation. PLoS One 2014;9:e115933

25. Marzi S, Piludu F, Sanguineti G, Marucci L, Farneti A, Terrenato $I$, et al. The prediction of the treatment response of cervical nodes using intravoxel incoherent motion diffusion-weighted imaging. Eur J Radiol 2017;92:93-102

26. Lombardi M, Cascone T, Guenzi E, Stecco A, Buemi F, Krengli $M$, et al. Predictive value of pre-treatment apparent diffusion coefficient $(A D C)$ in radio-chemiotherapy treated head and neck squamous cell carcinoma. Radiol Med 2017;122:345-352

27. Lambrecht M, Van Calster B, Vandecaveye V, De Keyzer F, Roebben I, Hermans $R$, et al. Integrating pretreatment diffusion weighted MRI into a multivariable prognostic model for head and neck squamous cell carcinoma. Radiother Oncol 2014;110:429-434

28. Kim S, Loevner L, Quon H, Sherman E, Weinstein G, Kilger $A$, et al. Diffusion-weighted magnetic resonance imaging for predicting and detecting early response to chemoradiation therapy of squamous cell carcinomas of the head and neck. Clin Cancer Res 2009;15:986-994

29. Hauser T, Essig M, Jensen A, Gerigk L, Laun FB, Munter $M$, et al. Characterization and therapy monitoring of head and neck carcinomas using diffusion-imaging-based intravoxel incoherent motion parameters-preliminary results. Neuroradiology 2013;55:527-536

30. Hatakenaka M, Shioyama Y, Nakamura K, Yabuuchi H, Matsuo Y, Sunami S, et al. Apparent diffusion coefficient calculated with relatively high b-values correlates with local failure of head and neck squamous cell carcinoma treated with radiotherapy. AJNR Am J Neuroradiol 2011;32:1904-1910

31. Hatakenaka M, Nakamura K, Yabuuchi H, Shioyama Y, Matsuo Y, Ohnishi K, et al. Pretreatment apparent diffusion coefficient of the primary lesion correlates with local failure in head-andneck cancer treated with chemoradiotherapy or radiotherapy. Int J Radiat Oncol Biol Phys 2011;81:339-345

32. Hatakenaka M, Nakamura K, Yabuuchi H, Shioyama Y, Matsuo $Y$, Kamitani $T$, et al. Apparent diffusion coefficient is a prognostic factor of head and neck squamous cell carcinoma treated with radiotherapy. Jpn J Radiol 2014;32:80-89

33. Vandecaveye V, Dirix P, De Keyzer F, Op de Beeck K, Vander Poorten V, Hauben E, et al. Diffusion-weighted magnetic resonance imaging early after chemoradiotherapy to monitor treatment response in head-and-neck squamous cell carcinoma. Int J Radiat Oncol Biol Phys 2012;82:1098-1107

34. King AD, Chow KK, Yu KH, Mo FK, Yeung DK, Yuan J, et al. Head and neck squamous cell carcinoma: diagnostic performance of diffusion-weighted MR imaging for the prediction of treatment response. Radiology 2013;266:531-538

35. Wong KH, Panek R, Welsh L, McQuaid D, Dunlop A, Riddell A, et al. The predictive value of early assessment after 1 cycle of induction chemotherapy with 18F-FDG PET/CT and diffusionweighted mri for response to radical chemoradiotherapy in head and neck squamous cell carcinoma. J Nucl Med 2016;57:1843-1850

36. Schouten CS, de Bree R, van der Putten L, Noij DP, Hoekstra OS, Comans EF, et al. Diffusion-weighted EPI- and HASTEMRI and 18F-FDG-PET-CT early during chemoradiotherapy in advanced head and neck cancer. Quant Imaging Med Surg 2014;4:239-250

37. Paudyal R, Oh JH, Riaz N, Venigalla P, Li J, Hatzoglou V, et al. Intravoxel incoherent motion diffusion-weighted MRI during chemoradiation therapy to characterize and monitor treatment response in human papillomavirus head and neck squamous cell carcinoma. J Magn Reson Imaging 2017;45:1013-1023

38. Galbán CJ, Mukherji SK, Chenevert TL, Meyer CR, Hamstra $\mathrm{DA}$, Bland $\mathrm{PH}$, et al. A feasibility study of parametric response map analysis of diffusion-weighted magnetic resonance imaging scans of head and neck cancer patients for providing early detection of therapeutic efficacy. Transl Oncol 2009;2:184-190

39. Aramburu Núñez D, Lopez MA, Mera IM, Salvador GF, Dave A, Hatzoglou $V$, et al. Multimodality functional imaging using DW-MRI and ${ }^{18} \mathrm{~F}-\mathrm{FDG}$-PET/CT during radiation therapy for human papillomavirus negative head and neck squamous cell carcinoma: Meixoeiro Hospital of Vigo Experience. World J Radiol 2017;9:17-26

40. Hauser T, Essig M, Jensen A, Laun FB, Munter M, MaierHein $\mathrm{KH}$, et al. Prediction of treatment response in head and neck carcinomas using IVIM-DWI: evaluation of lymph node metastasis. Eur J Radiol 2014;83:783-787

41. Whiting PF, Rutjes AW, Westwood ME, Mallett S, Deeks JJ, Reitsma JB, et al. QUADAS-2: a revised tool for the quality assessment of diagnostic accuracy studies. Ann Intern Med 2011;155:529-536

42. Deville WL, Buntinx F, Bouter LM, Montori VM, de Vet HC, van der Windt DA, et al. Conducting systematic reviews of diagnostic studies: didactic guidelines. BMC Med Res Methodol 2002;2:9

43. Vandecaveye V, Dirix P, De Keyzer F, de Beeck K0, Vander Poorten V, Roebben I, et al. Predictive value of diffusion-weighted magnetic resonance imaging during chemoradiotherapy for head and neck squamous cell carcinoma. Eur Radiol 2010;20:1703-1714

44. King AD, Thoeny HC. Functional MRI for the prediction of treatment response in head and neck squamous cell carcinoma: potential and limitations. Cancer Imaging 2016;16:23

45. Driessen JP, Caldas-Magalhaes J, Janssen LM, Pameijer FA, Kooij N, Terhaard CH, et al. Diffusion-weighted MR imaging in laryngeal and hypopharyngeal carcinoma: association between apparent diffusion coefficient and histologic findings. 
DW Imaging for Predicting Treatment Response in Squamous Cell Carcinoma

\section{Radiology 2014;272:456-463}

46. Driessen JP, van Bemmel AJ, van Kempen PM, Janssen LM, Terhaard CH, Pameijer FA, et al. Correlation of human papillomavirus status with apparent diffusion coefficient of diffusion-weighted MRI in head and neck squamous cell carcinomas. Head Neck 2016;38 Suppl 1:E613-E618

47. Nakahira M, Saito N, Yamaguchi H, Kuba K, Sugasawa M. Use of quantitative diffusion-weighted magnetic resonance imaging to predict human papilloma virus status in patients with oropharyngeal squamous cell carcinoma. Eur Arch Otorhinolaryngol 2014;271:1219-1225

48. Abdel Razek AA, Kandeel AY, Soliman N, El-shenshawy HM, Kamel $\mathrm{Y}, \mathrm{Nada} \mathrm{N}$, et al. Role of diffusion-weighted echoplanar MR imaging in differentiation of residual or recurrent head and neck tumors and posttreatment changes. AJNR Am J Neuroradiol 2007;28:1146-1152

49. Pickles MD, Gibbs P, Lowry M, Turnbull LW. Diffusion changes precede size reduction in neoadjuvant treatment of breast cancer. Magn Reson Imaging 2006;24:843-847

50. Uhl M, Saueressig U, van Buiren M, Kontny U, Niemeyer C, Kohler G, et al. Osteosarcoma: preliminary results of in vivo assessment of tumor necrosis after chemotherapy with diffusion- and perfusion-weighted magnetic resonance imaging. Invest Radiol 2006;41:618-623

51. Mardor Y, Pfeffer R, Spiegelmann R, Roth Y, Maier SE, Nissim 0 , et al. Early detection of response to radiation therapy in patients with brain malignancies using conventional and high b-value diffusion-weighted magnetic resonance imaging. $\mathrm{J}$ Clin Oncol 2003;21:1094-1100

52. Chen YG, Chen MQ, Guo YY, Li SC, Wu JX, Xu BH. Apparent diffusion coefficient predicts pathology complete response of rectal cancer treated with neoadjuvant chemoradiotherapy. PLoS One 2016;11:e0153944

53. Onal C, Erbay G, Guler OC. Treatment response evaluation using the mean apparent diffusion coefficient in cervical cancer patients treated with definitive chemoradiotherapy. J Magn Reson Imaging 2016;44:1010-1019

54. Argiris A, Stenson KM, Brockstein BE, Mittal BB, Pelzer H, Kies MS, et al. Neck dissection in the combined-modality therapy of patients with locoregionally advanced head and neck cancer. Head Neck 2004;26:447-455

55. Brizel DM, Prosnitz RG, Hunter S, Fisher SR, Clough RL, Downey MA, et al. Necessity for adjuvant neck dissection in setting of concurrent chemoradiation for advanced head-andneck cancer. Int J Radiat Oncol Biol Phys 2004;58:1418-1423

56. Lopez Rodriguez M, Cerezo Padellano L, Martin Martin M, Couñago Lorenzo F. Neck dissection after radiochemotherapy in patients with locoregionally advanced head and neck cancer. Clin Transl Oncol 2008;10:812-816

57. Le Bihan D, Breton E, Lallemand D, Aubin ML, Vignaud J, Laval-Jeantet M. Separation of diffusion and perfusion in intravoxel incoherent motion MR imaging. Radiology 1988;168:497-505 